\title{
LINKS AND SUBMERSIONS TO THE PLANE ON AN OPEN 3-MANIFOLD
}

\author{
SHIGEAKI MIYOSHI
}

\begin{abstract}
We study the realization problem which asks if a given oriented link in an open 3-manifold can be realized as a fiber of a submersion to the Euclidean plane. We correct the results obtained before by the author which contains an error and certain imperfection, and investigate a necessary and sufficient condition for the realization in the words of well-known invariants. We obtain the condition expressed by the first homology group with mod 2 coefficient.
\end{abstract}

\section{INTRODUCTION}

The purpose of this paper is two-fold. First, we will correct a theorem in $\mathrm{My}$ by the author, and second, study a problem which arises from the correction. G. Hector and D. Peralta-Salas found the error in the theorem in $\mathrm{My}$ and informed the author about it. Moreover, they studied comprehensively the realization problem which asks if a manifold can be embedded in another manifold so that it is also a fiber of a submersion to Euclidean space (see [HP]).

Before stating the correct theorem, we prepare some notions. We mostly work in the smooth $\left(C^{\infty}\right)$ category in this paper. Suppose that $M$ is an open oriented 3-manifold and $L$ is an oriented $n$-component link in $M$. In this paper we say that a manifold is open if the boundary is empty and no component is compact. Let $N(L)$ denote a small tubular neighborhood of $L$. A framing $\nu$ of $L$ is meant to be an embedding $\nu: \bigsqcup_{j=1}^{n}\left(S^{1} \times D^{2}\right)_{j} \rightarrow M$ onto $N(L)$ which maps the cores $\bigsqcup_{j=1}^{n}\left(S^{1} \times\{0\}\right)_{j}$ onto $L=\bigsqcup_{j=1}^{n} L_{j}$. Here, $D^{2}$ denotes the unit disk in $\mathbb{C}$ and $S^{1}=\partial D^{2}$. We assume that any framings

2000 Mathematics Subject Classification. primary 57R30, secondary 57R99, 57M99, $57 \mathrm{R} 25$.

Key words and phrases. knot, submersion, completely integrable vector field.

The author is supported by Grant-in-Aid for Scientific Research 23540106. 
and their restrictions to the cores are orientation-preserving. We note that a framing of $L$ induces a tangential framing of $T N(L)(=T M \mid N(L))$ and vice versa. Here, a tangential framing means a choice of a trivialization of $T N(L) \cong N(L) \times\left(\mathbb{R} \times \mathbb{R}^{2}\right)$ with $T N(L) \mid L=T L \oplus T L^{\perp} \cong(L \times \mathbb{R}) \oplus\left(L \times \mathbb{R}^{2}\right)$ where $T L^{\perp}$ denotes a normal bundle to $T L$.

Definition. Suppose that $L$ represents the null-class in the locally finite homology group $H_{1}^{\infty}(M ; \mathbb{Z})$, i.e., the homology group of locally finite (possibly) infinite chains. A framing $\nu$ of $L$ is said to be preferred (or null-homologous) if the union of the longitudes $\nu\left(\bigsqcup_{j=1}^{n}\left(S^{1} \times\{1\}\right)_{j}\right)$ represents the null-class in $H_{1}^{\infty}(M \backslash \operatorname{Int} N(L) ; \mathbb{Z})$. We call $\nu\left(\bigsqcup_{j=1}^{n}\left(S^{1} \times\{1\}\right)_{j}\right)$ the preferred longitudes of $N(L)$ with respect to the preferred framing $\nu$.

Remark 1. If $L$ represents the null-class in $H_{1}^{\infty}(M ; \mathbb{Z})$, then there exists a preferred framing of $L$. In fact, there exists an oriented (possibly noncompact) surface in $M$ bounded by $L$. Choosing such a surface $S$, we have a framing of $L$ whose longitudes are $S \cap \partial N(L)$.

Remark 2. Note that a preferred framing is not unique in general. In fact, in the case of the core circle of the open solid torus, every framing is preferred. Nevertheless, we invoke the terminology of preferred longitudes in $[\mathrm{R}]$.

The correct theorem is the following.

Theorem A. For an oriented link $L$ in an open oriented 3 -manifold $M$, the following conditions are equivalent:

(1) there exists a submersion $\varphi: M \rightarrow \mathbb{R}^{2}$ such that up to isotopy the preimage $\varphi^{-1}(0)$ of the origin is $L$ and $\varphi$ maps the transverse orientation of $L$ to the standard orientation of $\mathbb{R}^{2}$, i.e., for any small disk $D$ transverse to $L$ with the orientation induced from those of $M$ and $L$, the restriction $\varphi \mid D$ preserves the orientation, and

(2) the cycle $L$ represents the null-class in the locally finite homology group $H_{1}^{\infty}(M ; \mathbb{Z})$ and there exists a preferred framing of $L$ whose tangential framing is the restriction of some trivialization of TM. 
Theorem $\mathrm{A}$ is also a consequence of Theorem 2.4.2 in $[\mathrm{HP}]$. In the original incorrect theorem (Theorem 1 in $[\mathrm{My}]$ ) the above extension condition of the framing in (2) is missing. Here, we explain briefly how it fills the gap in the original proof. If (1) holds, then the canonical trivialization of the tangent bundle of $\mathbb{R}^{2}$ is pulled back to a normal bundle to the fibers. With a trivialization of the tangent bundle to the fibers, it determines a trivialization of $T M$ which restricts to a tangential framing of $L$. The projection map from $N(L)$ onto the meridian disk determined by this framing must coincide with the submersion restricted to $N(L)$. Conversely, by the assumption that the framing of $L$ is preferred, the projection map $N(L) \approx \bigsqcup_{j=1}^{n}\left(S^{1} \times D^{2}\right)_{j} \rightarrow D^{2}$ extends to a map $(M, M \backslash \operatorname{Int} N(L)) \rightarrow\left(\mathbb{R}^{2}, \mathbb{R}^{2} \backslash \operatorname{Int} D^{2}\right)$ and moreover an extension of the (tangential) framing of $L$ to $M$ ensures that we can take a submersion $M \rightarrow \mathbb{R}^{2}$ as the extended map. This is an application of the h-principle, in this case, A. Phillips' submersion classification theory $[\mathrm{P}]$. In the proof in $\mathrm{My}$, it is only shown that an extension as a map exists since the framing of $L$ is preferred. However, in order to apply Phillips' theory to have an extended submersion, we need the requirement of the simultaneous extension of the tangential framing of $L$ and the projection map on $N(L)$ to the whole manifold $M$.

We note that Theorem 2 in $\mathrm{My}$ is correct even though the proof in $\mathrm{My}$ is not completed.

Theorem B (Theorem 2 in $[\mathrm{My}]$ ). For any link $L$ in an open orientable 3-manifold, there is a submersion $\varphi: M \rightarrow \mathbb{R}^{2}$ such that up to isotopy the union of compact components of $\varphi^{-1}(0)$ is $L$.

In order to prove this theorem, we have to choose a (tangential) framing of $L$ which is the restriction of some trivialization over the whole manifold $M$. This can be always done by twisting a framing once around the meridional direction if necessary. Note that we need not to require that the framing is preferred here. This observation is missing in the proof in $\mathrm{My}$. Theorem $\mathrm{B}$ is also proved in Application 2.3.8 in [HP]. We will give the proof of Theorem $\mathrm{A}$ and $\mathrm{B}$ in Section 4 as an appendix. 
As a consequence of the correction, there arises a question to find a criterion for a link to be a fiber of a submersion to the plane in the words of well-known invariants. We will answer to this question for the case of a knot. Suppose that $M$ is an open oriented 3-manifold and $K$ is an oriented knot in $M$. For the simplicity, we say that $K$ is realizable if $K$ satisfies the condition (11) in Theorem A,

The following is the main theorem of this paper.

Main Theorem. Assume that $K$ represents the null-class in $H_{1}^{\infty}(M ; \mathbb{Z})$. Then, $K$ is realizable if and only if $K$ represents a non-zero class in $H_{1}\left(M ; \mathbb{Z}_{2}\right)$, where $\mathbb{Z}_{2}=\mathbb{Z} / 2 \mathbb{Z}$.

In order to prove the Main Theorem, it suffices to show the following two claims. Let $\kappa$ denote the homology class $\iota_{*}([K]) \in H_{1}(M ; \mathbb{Z})$, where $\iota: K \hookrightarrow$ $M$ is the inclusion map and $[K]$ denotes the fundamental class of $K$. Also, let $\kappa_{(2)}$ denote the $\mathbb{Z}_{2}$-reduction of $\kappa$ in $H_{1}\left(M ; \mathbb{Z}_{2}\right)$.

Claim 1. If $\kappa_{(2)} \neq 0$ then $K$ is realizable.

Claim 2. If $\kappa_{(2)}=0$ then $K$ is not realizable.

Remark 3. As mentioned earlier, G. Hector and D. Peralta-Salas [HP] studied this kind of realization problem in the more general dimensions and setting. As one application of their theory, they obtained a characterization for a link in $\mathbb{R}^{3}$ to be realizable and in particular they showed that no knot in $\mathbb{R}^{3}$ is realizable. One may consider that the Main Theorem generalizes the result.

Remark 4. In the case of links, the argument will be a rather complicated nuisance. It might be just a technicality, nevertheless we omit here the consideration in the case of links at all. The complete research including the general case of links should be done in the sequel.

In Section 2, we describe the notion of tangential framings of oriented knots from the homotopical viewpoint, and prove Claim 1. In Section 3, we study the properties of framings of oriented knots and prove Claim 2, For 
the reader's convenience, we state a part of Phillips' theory $[\mathrm{P}]$ which we need and give the proofs of Theorem $\mathrm{A}$ and $\mathrm{B}$ in Section 4 , as an appendix.

\section{Proof of Claim 1}

First, we express the notion of framings of oriented knots in different words. Suppose that $M$ is an oriented open 3-manifold and $K$ is an oriented knot in $M$. We fix a trivialization $\Pi: T M \cong M \times \mathbb{R}^{3}$ throughout the paper. Suppose any framing $\nu: S^{1} \times D^{2} \rightarrow N(K)$ is given. Isotoping $\nu$ if necessary, we may assume that each meridian disk $\nu\left(\{\exp t \sqrt{-1}\} \times D^{2}\right)$ is normal to $K$ with respect to the metric induced by $\Pi$. We will define a map $f_{\nu}: K \rightarrow \mathrm{SO}(3)$ which is an alternate of $\nu$ under $\Pi$ as follows. For any point $p \in K$, let $\left(v_{1}(p), v_{2}(p), v_{3}(p)\right)$ be the orthonormal frame of $T_{p} M=\mathbb{R}^{3}$ determined by the tangent bundle and the normal bundle to $K$. Precisely, $v_{1}(p)$ is the unit tangent vector to $K, v_{2}(p)$ is the unit normal vector determined by $\frac{\partial}{\partial x}$, and another unit normal vector $v_{3}(p)$ is chosen by the orientation of $M$. Here, we write $z=x+y \sqrt{-1} \in D^{2}$. Thus, with respect to $\Pi$, this orthonormal frame $\left(v_{1}(p), v_{2}(p), v_{3}(p)\right)$ can be expressed as a special orthogonal matrix. We define $f_{\nu}(p):=\left(v_{1}(p), v_{2}(p), v_{3}(p)\right) \in \mathrm{SO}(3)$.

Definition. We call the resulting map $f_{\nu}: K \rightarrow \mathrm{SO}(3)$ a $\sigma$-framing of $K$ with respect to $\nu$.

Remark 5. In fact, a $\sigma$-framing is the component of a cross section of the frame bundle $\operatorname{Fr}(T M) \cong M \times \mathrm{SO}(3)$ associated with $T M$ with the trivialization induced by $\Pi$.

It can be easily seen that under the parallelization $\Pi$ any map $K \rightarrow \mathrm{SO}(3)$ determines a framing $S^{1} \times D^{2} \rightarrow N(K)$ up to isotopy. Thus, to choose a framing (up to isotopy) and to choose a $\sigma$-framing (up to homotopy) are equivalent. Moreover, since $\left[S^{1}, \mathrm{SO}(3)\right] \cong \operatorname{Hom}\left(\pi_{1}\left(S^{1}\right), \pi_{1}(\mathrm{SO}(3))\right) \cong$ $\pi_{1}(\mathrm{SO}(3)) \cong H_{1}(\mathrm{SO}(3) ; \mathbb{Z}) \cong \mathbb{Z}_{2}$ with appropriate choices of base points, we may identify the homotopy class $\left[f_{\nu}\right]$ of a $\sigma$-framing with its image $\left(f_{\nu}\right)_{*}([K]) \in H_{1}(\mathrm{SO}(3) ; \mathbb{Z}) \cong \pi_{1}(\mathrm{SO}(3))$. Note that a tangential framing of $K$ is the restriction of some trivialization of $T M$ if and only if the corresponding $\sigma$-framing of $K$ extends to $M$ as a map. 
Now we have the following criteria for the existence of an extension of a $\sigma$-framing.

Lemma 1. Let $M$ be an open orientable 3-manifold and $K$ a knot in $M$. Suppose that a map $f: K \rightarrow \mathrm{SO}(3)$ is given. Then the following are equivalent:

(1) the map $f: K \rightarrow \mathrm{SO}(3)$ extends to a map $M \rightarrow \mathrm{SO}(3)$,

(2) the induced homomorphism $f_{*}: \pi_{1}(K) \rightarrow \pi_{1}(\mathrm{SO}(3))$ extends to a homomorphism $\pi_{1}(M) \rightarrow \pi_{1}(\mathrm{SO}(3))$, and

(3) the homomorphism $f_{*}: H_{1}(K ; \mathbb{Z}) \rightarrow H_{1}(\mathrm{SO}(3) ; \mathbb{Z})$ extends to a homomorphism $H_{1}(M ; \mathbb{Z}) \rightarrow H_{1}(\mathrm{SO}(3) ; \mathbb{Z})$.

Moreover, in the implication from (2) or (3) to (1), the resulting extension map $M \rightarrow \mathrm{SO}(3)$ induces the given extended homomorphism.

Proof. It is well known that an open orientable 3-manifold is homotopy equivalent to a subcomplex of its 2-skeleton (cf. [P], [W] for example). Therefore, by an elementary obstruction theory, the given map $f: K \rightarrow$ $\mathrm{SO}(3)$ extends to a map $M \rightarrow \mathrm{SO}(3)$ if and only if the induced homomorphism $f_{*}: \pi_{1}(K) \rightarrow \pi_{1}(\mathrm{SO}(3))$ extends to a homomorphism $\pi_{1}(M) \rightarrow$ $\pi_{1}(\mathrm{SO}(3))$, i.e., there is a homomorphism $\Phi: \pi_{1}(M) \rightarrow \pi_{1}(\mathrm{SO}(3))$ such that $\Phi \circ \iota_{*}=f_{*}$ where $\iota: K \hookrightarrow M$ is the inclusion. Moreover, since $\pi_{1}(K)$ and $\pi_{1}(\mathrm{SO}(3))$ are Abelian it is equivalent to the condition that the homomorphism $f_{*}: H_{1}(K ; \mathbb{Z}) \rightarrow H_{1}(\mathrm{SO}(3) ; \mathbb{Z})$ extends to a homomorphism $H_{1}(M ; \mathbb{Z}) \rightarrow H_{1}(\mathrm{SO}(3) ; \mathbb{Z})$.

Now, we show Claim 1 .

Proof of Claim 1. Suppose $f: K \rightarrow \mathrm{SO}(3)$ is a preferred $\sigma$-framing of $K$, i.e., the $\sigma$-framing associated with a preferred framing of $K$. By Theorem $\mathrm{A}$ and Lemma 1, it suffices to show that there exists a homomorphism $\Phi: H_{1}(M ; \mathbb{Z}) \rightarrow H_{1}(\mathrm{SO}(3) ; \mathbb{Z})$ which is an extension of the induced homomorphism $f_{*}: H_{1}(K ; \mathbb{Z}) \rightarrow H_{1}(\mathrm{SO}(3) ; \mathbb{Z})$. If $f_{*}=0$ then the zero homomorphism is an extension. Hence we assume $f_{*} \neq 0$, which implies $f_{*}$ is an epimorphism. On the other hand, since $\kappa_{(2)} \neq 0$ the composition of the natural homomorphisms $H_{1}(K ; \mathbb{Z}) \rightarrow H_{1}(M ; \mathbb{Z}) \rightarrow H_{1}\left(M ; \mathbb{Z}_{2}\right)$ 
is non-trivial. Let $\left\langle\kappa_{(2)}\right\rangle$ denote its image. Since $H_{1}\left(M ; \mathbb{Z}_{2}\right)$ is a vector space over the field $\mathbb{Z}_{2}$, we have a projection onto the one-dimensional subspace $H_{1}\left(M ; \mathbb{Z}_{2}\right) \rightarrow\left\langle\kappa_{(2)}\right\rangle$. Let $\Psi$ denote the composition of the natural homomorphism $H_{1}(M ; \mathbb{Z}) \rightarrow H_{1}\left(M ; \mathbb{Z}_{2}\right)$ followed by this projection. Let $\alpha:\left\langle\kappa_{(2)}\right\rangle \cong \mathbb{Z}_{2}$ and $\beta: H_{1}(\mathrm{SO}(3) ; \mathbb{Z}) \cong \mathbb{Z}_{2}$ be any isomorphisms. Then the composition $\Phi=\beta^{-1} \circ \alpha \circ \Psi$ is the desired extension homomorphism.

\section{Lemmata and proof of Claim 2}

In this section, we study some properties of $\sigma$-framings and prove Claim 2. The following lemma describes a relation of $(\sigma-)$ framings of two oriented knots which are homologous.

Lemma 2. Let $Z_{1}$ and $Z_{2}$ be oriented knots in $M$ and $\nu_{j}: S^{1} \times D^{2} \rightarrow N\left(Z_{j}\right)$ their framings $(j=1,2)$. Assume that there is a compact oriented surface $S$ in $M$ such that $\partial S=Z_{1} \sqcup\left(-Z_{2}\right)$ and $S \cap \partial N\left(Z_{j}\right)=\nu_{j}\left(S^{1} \times\{1\}\right)$, where $-Z_{2}$ denotes $Z_{2}$ with the orientation reversed. Then the induced $\sigma$-framings $f_{\nu_{1}}$ and $f_{\nu_{2}}$ satisfy that $\left[f_{\nu_{1}}\right]=\left[f_{\nu_{2}}\right] \in \pi_{1}(\mathrm{SO}(3))$.

Proof of Lemma 2. We define a map $F: S \rightarrow \mathrm{SO}(3)$ as follows. Choose a unit tangent vector field $v_{1}: S \rightarrow T S \subset T M \mid S$ such that $v_{1} \mid Z_{1}$ coincides with the unit vector field tangent to $Z_{1}$. Then we choose another vector field $v_{2}$ so that $\left(v_{1}, v_{2}\right)$ forms an orthonormal frame field of $S$. Here, $v_{2}$ is chosen to be inward normal along $Z_{1}$. Picking the normal unit vector field $v^{\perp}$ to $S$, we have a frame field $F=\left(v_{1}, v_{2}, v^{\perp}\right): S \rightarrow \mathrm{SO}(3)$. By the definition, $F \mid Z_{1}=f_{\nu_{1}}$. Since the rotation number of $v_{1} \mid Z_{2}$ along $Z_{2}$ is equal to the Euler characteristic $\chi(S)$ which is the minus twice the genus of $S$, we have $\left[F \mid Z_{2}\right]=\left[f_{\nu_{2}}\right] \in \pi_{1}(\mathrm{SO}(3))$. Since $F \mid Z_{1}$ and $F \mid Z_{2}$ are homologous (or bordant) by $F$, we have $\left[f_{\nu_{1}}\right]=\left[F \mid Z_{1}\right]=\left[F \mid Z_{2}\right]=\left[f_{\nu_{2}}\right]$.

Next, we study an oriented knot whose homology class with $\mathbb{Z}_{2}$ coefficient is zero. First, we consider the "double" of a knot and study its framing. Let $J$ be any oriented knot in $M$ and $\nu: S^{1} \times D^{2} \rightarrow N(J)$ any framing of $J$. Let $J_{d}$ be the $(2,1)$-cable knot in $N(J)$. For the clarity, we define $J_{d}$ as follows. 
Define $\tilde{L}$ to be a union of two parallel lines in $\mathbb{R} \times D^{2}$ as

$$
\tilde{L}:=\left\{\left(t, \pm \frac{1}{2}\right) \mid t \in \mathbb{R},\right\}
$$

and $\tau$ to be a self-diffeomorphism on $\mathbb{R} \times D^{2}$ by $\tau(t, z):=(t, \exp (\pi t \sqrt{-1}) z)$. Then the quotient of $\left(\mathbb{R} \times D^{2}, \tau(\tilde{L})\right)$ by the $\mathbb{Z}$-action generated by the translation by 1 on the $\mathbb{R}$-factor is a manifold pair $\left(S^{1} \times D^{2}, L\right)$ such that $L$ is an oriented knot. Here, we identify $S^{1}=\mathbb{R} / \mathbb{Z}$. We define $J_{d}$ to be $\nu(L)$ and call it a $(2,1)$-cable knot of $J$ with respect to $\nu$.

To define a natural framing of a $(2,1)$-cable knot $J_{d}$ of $J$, we consider an annulus in $S^{1} \times D^{2}$ defined as follows. Let $\tilde{A}$ be a union of two strips in $\mathbb{R} \times D^{2}$ defined by

$$
\tilde{A}:=\left\{(t, \pm r) \mid t \in \mathbb{R}, \frac{1}{2} \leq r \leq 1\right\}
$$

and set $\left(S^{1} \times D^{2}, A\right):=\left(\mathbb{R} \times D^{2}, \tau(\tilde{A})\right) / \mathbb{Z}$ as the quotient by the translation. Note that $\partial A-\partial\left(S^{1} \times D^{2}\right)=L$. For any small tubular neighborhood $N(L)$ in $\operatorname{Int}\left(S^{1} \times D^{2}\right)$, there is a framing $\xi: S^{1} \times D^{2} \rightarrow N(L) \subset S^{1} \times D^{2}$ of $L$ such that $\xi\left(S^{1} \times\{1\}\right)=\partial N(L) \cap A$. We call the framing $\nu \circ \xi: S^{1} \times D^{2} \rightarrow N\left(J_{d}\right)=$ $\nu(N(L)) \subset N(J)$ a revolution framing of the $(2,1)$-cable knot $J_{d}(=\nu(L))$ of $J$ with respect to $\nu$. See Figure 1. Under the parallelization $\Pi$ of $M$, the

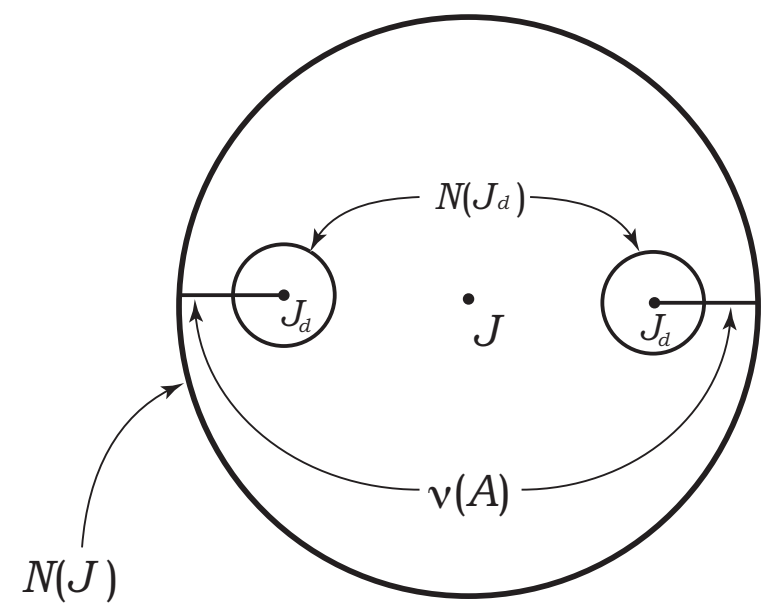

Figure 1. A revolution framing of $J_{d}$ at a section

revolution framing of $J_{d}$ induces a revolution $\sigma$-framing $J_{d} \rightarrow \mathrm{SO}(3)$.

The following is a key lemma to the proof of Claim 2 , 
LINKS AND SUBMERSIONS TO THE PLANE ON AN OPEN 3-MANIFOLD 9

Lemma 3. For any oriented knot $J$ in $M$ and any framing $\nu: S^{1} \times D^{2} \rightarrow$ $N(J)$, the revolution $\sigma$-framing $f_{d}: J_{d} \rightarrow \mathrm{SO}(3)$ of the $(2,1)$-cable knot $J_{d}$ with respect to $\nu$ is not null-homotopic, i.e., $\left[f_{d}\right]=1 \in \mathbb{Z}_{2} \cong \pi_{1}(\mathrm{SO}(3))$.

Proof. Let $f_{\nu}: J \rightarrow \mathrm{SO}(3)$ be the $\sigma$-framing of $J$ with respect to $\nu$. Then along $J_{d}$ the frame $f_{d}(p)$ goes around twice in the longitudinal direction and rotates once in the meridional direction. Thus $\left[f_{d}\right]=2\left[f_{\nu}\right]+1 \equiv 1 \in \mathbb{Z}_{2} \cong$ $\pi_{1}(\mathrm{SO}(3))$.

Next, suppose that $K$ is an oriented knot $K$ which represents the null-class in $H_{1}^{\infty}(M ; \mathbb{Z})$. Recall that $\kappa_{(2)} \in H_{1}\left(M ; \mathbb{Z}_{2}\right)$ denotes the $\mathbb{Z}_{2}$-reduction of the homology class $\kappa=\iota_{*}([K]) \in H_{1}(M ; \mathbb{Z})$. Let $\lambda$ be the homology class in $H_{1}(M \backslash \operatorname{Int} N(K) ; \mathbb{Z})$ represented by a preferred longitude of $N(K)$. Then we have the following.

Lemma 4. If $\kappa_{(2)}=0$, then the $\mathbb{Z}_{2}$-reduction $\lambda_{(2)}$ of $\lambda$ is zero.

Proof. Let $E_{M}(K)$ denote the knot exterior $M \backslash \operatorname{Int} N(K)$. Consider the following diagram.

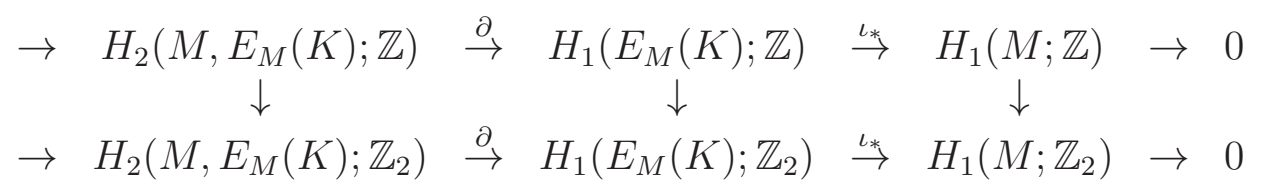

Here, the rows are homology exact sequences of the pair $\left(M, E_{M}(K)\right)$ and the vertical arrows are natural homomorphisms. Then $\iota_{*}(\lambda)=\kappa, \iota_{*}\left(\lambda_{(2)}\right)=\kappa_{(2)}$ and $\lambda, \kappa$ are mapped down to $\lambda_{(2)}, \kappa_{(2)}$ respectively. Since $\kappa_{(2)}=0$, there is $\eta \in H_{2}\left(M, E_{M}(K) ; \mathbb{Z}_{2}\right)$ such that $\partial \eta=\lambda_{(2)}$. Note that $H_{2}\left(M, E_{M}(K) ; \mathbb{Z}_{2}\right)$ is isomorphic to $\mathbb{Z}_{2}$ generated by the meridian disk of $N(K)$. Hence $\partial \eta$ is represented by the meridian loop or equal to zero. However, the meridian loop intersects exactly once with a (locally finite) relative cycle in $\left(E_{M}(K), \partial E_{M}(K)\right)$ bounded by the preferred longitude of $N(K)$, the representative cycle of $\lambda$. Thus $\partial \eta=\lambda_{(2)}$ must be zero.

The following is another key lemma which describes a normal form of the knot which satisfies the hypothesis of Claim 2 . 
Lemma 5. Suppose that an oriented knot $K$ represents the null-class in $H_{1}^{\infty}(M ; \mathbb{Z})$ and $\kappa_{(2)}=0$. We fix a preferred framing $\nu: S^{1} \times D^{2} \rightarrow N(K)$. Then, there exists an oriented knot $Z \subset M$ such that the $(2,1)$-cable knot $Z_{d}$ of $Z$ is homologous to $K$ and $\left[f_{d}\right]=\left[f_{\nu}\right] \in \pi_{1}(\mathrm{SO}(3))$, where $f_{d}: Z_{d} \rightarrow \mathrm{SO}(3)$ is the revolution $\sigma$-framing of $Z_{d}$ with respect to some framing of $Z$.

Proof. Let $L$ denote the preferred longitude of $N(K)$ with respect to $\nu$ and $\lambda$ the homology class in $H_{1}\left(E_{M}(K) ; \mathbb{Z}\right)$ represented by $L$. Then we have $\lambda_{(2)}=0$ by Lemma 4 using the assumption $\kappa_{(2)}=0$. Considering the Bockstein homology exact sequence with respect to $0 \rightarrow \mathbb{Z} \stackrel{\times 2}{\rightarrow} \mathbb{Z} \rightarrow \mathbb{Z}_{2} \rightarrow 0$, we have $\lambda=2 \zeta$ for some $\zeta \in H_{1}\left(E_{M}(K) ; \mathbb{Z}\right)$. Choose a representative cycle (an oriented knot) $Z \subset E_{M}(K)$ of $\zeta$ and a framing of $Z$. Since $\lambda=2 \zeta$, there exists an immersed oriented surface bounded by $L$ and "twice of $-Z$ ". Precisely, there exists an immersion $h$ of compact oriented surface $S$ into $E_{M}(K)$ which maps Int $S$ into Int $E_{M}(K)$ with the following properties. The boundary $\partial S$ is decomposed into two parts: $\partial_{+} S \sqcup \partial_{-} S$, where $h\left(\partial_{+} S\right)=L$ and $h\left(\partial_{-} S\right)=-Z$ which means $h \mid \partial_{-} S$ is orientation-reversing. The immersion $h$ is an embedding away from $\partial_{-} S$ and $h \mid \partial_{-} S$ is a (possibly trivial) two-fold covering onto $Z$. Moreover, $h(S) \cap N(Z)$ is homeomorphic (in fact diffeomorphic away from $Z$ ) to the mapping cylinder of the two-fold covering map $h \mid \partial_{-} S$. We may assume that $h(S)$ and $\partial N(Z)$ are transverse to each other and $h(S) \cap \partial N(Z)$ is a circle or a union of two parallel circles in $\partial N(Z)$. By choosing another framing of $N(Z)$ if necessary, we may assume that $h(S) \cap \partial N(Z)$ is the $(2,1)$-curve or a union of two $(1,0)$-curves with respect to the chosen framing of $N(Z)$ restricted to $\partial N(Z)$.

If $h(S) \cap \partial N(Z)$ is the $(2,1)$-curve, then attaching $A$ to $h(S)-\operatorname{Int} N(Z)$ along $h(S) \cap \partial N(Z)$ we have an embedded surface in $E_{M}(K)$ bounded by $L$ and $-Z_{d}$. Here, $A$ is the annulus in $N(Z)$ bounded by a $(2,1)$-curve in $\partial N(Z)$ and $Z_{d}$, which is defined in the definition of the revolution framing of $Z_{d}$, and the orientation of $A$ is determined by $-Z_{d}$. Since $L$ is isotopic to $K$ in $N(K)$, we have a compact oriented surface in $M$ bounded by $K$ and $-Z_{d}$. It follows that $K$ and $Z_{d}$ are homologous and $\left[f_{\nu}\right]=\left[f_{d}\right]$ by Lemma 2 . 
In the case that $h(S) \cap \partial N(Z)$ is two $(1,0)$-curves, we modify $h(S)$ in $N(Z)$ as follows. Consider the concentric tubular neighborhood $N_{1 / 2}(Z) \subset N(Z)$ where meridian disks are of radius $\frac{1}{2}$ of the meridian disks of $N(Z)$. We set a $(2,1)$-cable knot $Z_{d}$ on $\partial N_{1 / 2}(Z)$ with respect to the framing of $N(Z)$ and we will construct a compact oriented surface $B$ in $N(Z)$ bounded by $h(S) \cap \partial N(Z)$ and another longitude of $N\left(Z_{d}\right)$.
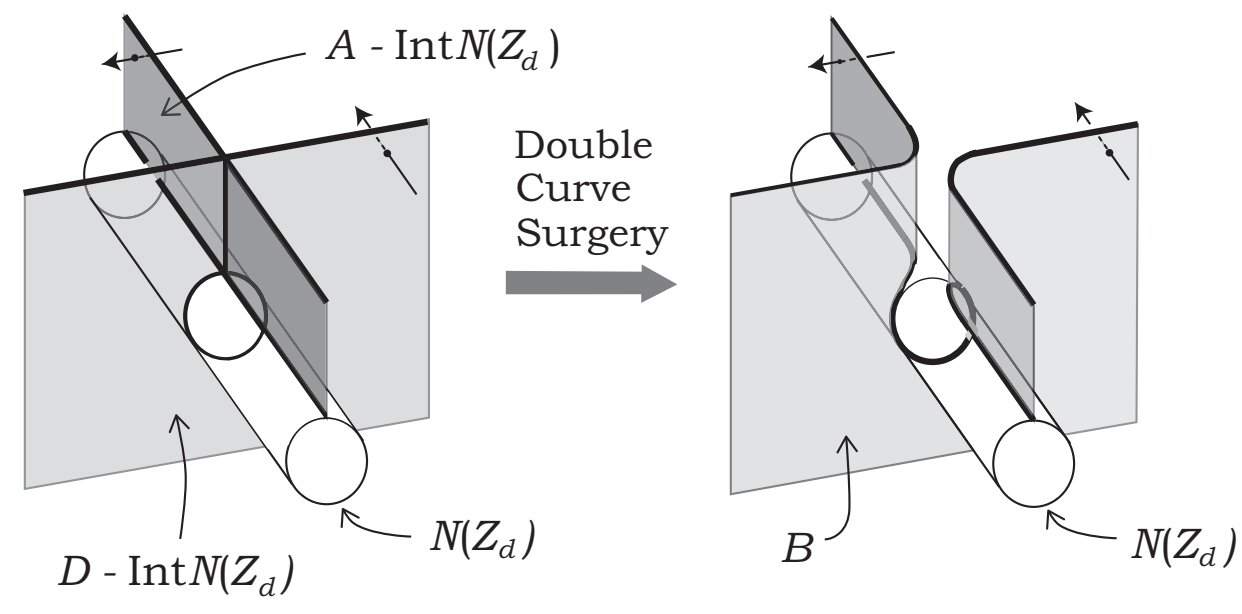

FiguRE 2. Double curve surgery on $(A \cup(-D))-\operatorname{Int} N\left(Z_{d}\right)$

First, let $D$ be a meridian disk of $N(Z)$ with the orientation induced from $S^{1} \times D^{2}$ by the framing and $A$ the annulus in $N(Z)$ which determines the revolution framing of $Z_{d}$ as above. Fix a small tubular neighborhood $N\left(Z_{d}\right)$ of $Z_{d}$. Then performing a double curve surgery on $(A \cup(-D))-\operatorname{Int} N\left(Z_{d}\right)$, we obtain an oriented surface $B$ (see Figure 2). By the construction, $B \cap$ $\partial N(Z)$ is a union of two $(1,0)$-curves and $B \cap \partial N\left(Z_{d}\right)$ is the longitude of the revolution framing with two twists corresponding to two intersection points between $D$ and $Z_{d}$. Since on $\partial N(Z)$ two curves $h(S) \cap \partial N(Z)$ and $B \cap \partial N(Z)$ are isotopic, we can attach $B$ to $h(S)-\operatorname{Int} N(Z)$ along $h(S) \cap \partial N(Z)$. Let $\Sigma$ denote the resulting surface: $\Sigma=(h(S)-\operatorname{Int} N(Z)) \cup B$. Then $\Sigma$ is a compact oriented proper surface in $E_{M}(K) \backslash \operatorname{Int} N\left(Z_{d}\right)$ and $\partial \Sigma=L \sqcup\left(-B \cap \partial N\left(Z_{d}\right)\right)$. Since $L$ (resp. $B \cap \partial N\left(Z_{d}\right)$ ) is isotopic to $K$ (resp. $Z_{d}$ ) in $N(K)$ (resp. $\left.N\left(Z_{d}\right)\right), K$ and $Z_{d}$ are homologous. On the other hand, the framing $\xi$ : $S^{1} \times D^{2} \rightarrow N\left(Z_{d}\right)$ such that $\xi\left(S^{1} \times\{1\}\right)=-\Sigma \cap \partial N\left(Z_{d}\right)\left(=-B \cap \partial N\left(Z_{d}\right)\right)$ 
determines a $\sigma$-framing $f_{\xi}: Z_{d} \rightarrow \mathrm{SO}(3)$. By Lemma 2, we have $\left[f_{\nu}\right]=\left[f_{\xi}\right]$. Moreover, as noted above, the longitude with respect to $\xi$ is the longitude of the revolution framing with two meridional twists. Hence we have $\left[f_{\xi}\right]=\left[f_{d}\right]$. Consequently, we have $\left[f_{d}\right]=\left[f_{\nu}\right]$.

Now we can prove Claim 2 .

Proof of Claim 2. Suppose that $f: K \rightarrow \mathrm{SO}(3)$ is any preferred $\sigma$-framing of $K$. In view of Theorem $\mathrm{A}$ and Lemma 1 , we only have to show that the induced homomorphism $f_{*}: H_{1}(K ; \mathbb{Z}) \rightarrow H_{1}(\mathrm{SO}(3) ; \mathbb{Z})$ never extends to $H_{1}(M ; \mathbb{Z})$. On the contrary to the conclusion, we assume that there is a homomorphism $\Phi: H_{1}(M ; \mathbb{Z}) \rightarrow H_{1}(\mathrm{SO}(3) ; \mathbb{Z})$ such that $\Phi \circ \iota_{*}=f_{*}$, where $\iota: K \hookrightarrow M$ denotes the inclusion. Since $\kappa_{(2)}=0$, we have $\Phi(\kappa)=0$. On the other hand, by Lemma 3 and $\left[5, f_{*}([K])=[f]=1\right.$. Therefore, we have $0=\Phi(\kappa)=\Phi \circ \iota_{*}([K])=f_{*}([K])=1$, a contradiction.

\section{Appendix: Proofs of Theorem A and B}

In order to prove Theorem $\mathrm{A}$ and $\mathrm{B}$, we review Phillips' submersion classification theory $[\mathrm{P}$. Let $X$ and $Y$ be manifolds. We assume that $\operatorname{dim} X \geq \operatorname{dim} Y$ in the following. The space of all submersions from $X$ to $Y$ is denoted by $\operatorname{Sbm}(X, Y)$ and the space of all vector bundle morphisms from $T X$ to $T Y$ whose restriction to each fiber the maximal rank by $\operatorname{Max}(T X, T Y)$. Here, $\operatorname{Sbm}(X, Y)$ and $\operatorname{Max}(T X, T Y)$ are endowed with $C^{1}$-compact-open topology and $C^{0}$-compact-open topology, respectively. If $X$ has a non-empty boundary, we impose no other condition on the boundary. The essence of the Phillips' theory is the following theorem.

Theorem 4.1 (Phillips $[\mathrm{P}]$ ). If $X$ has a handle decomposition with (possibly countably infinitely many) handles of indices less than $\operatorname{dim} X$, then the differential map d: $\operatorname{Sbm}(X, Y) \rightarrow \operatorname{Max}(T X, T Y)$ is a weak homotopy equivalence.

Since an open manifold has a handle decomposition with (countably infinitely many) handles of indices less than the dimension of the manifold, we have the following theorem as a corollary. 
Theorem 4.2 (Phillips $[\mathrm{P}]$ ). If $X$ is an open manifold, then the differential map d: $\operatorname{Sbm}(X, Y) \rightarrow \operatorname{Max}(T X, T Y)$ is a weak homotopy equivalence.

In the proof of Theorem 4.1 (and 4.2), the following are key lemmata.

Lemma 4.3. The differential map $d: \operatorname{Sbm}(D, Y) \rightarrow \operatorname{Max}(T D, T Y)$ is a weak homotopy equivalence, where $D$ denotes a disk of dimension $\operatorname{dim} X$.

Lemma 4.4. Let $V$ be a compact manifold with $\operatorname{dim} V=\operatorname{dim} X$. Suppose $W$ is obtained by attaching a handle of index less than $\operatorname{dim} V$. Then, the restriction maps $\rho: \operatorname{Sbm}(W, Y) \rightarrow \operatorname{Sbm}(\mathrm{V}, \mathrm{Y})$ and $\rho: \operatorname{Max}(T W, T Y) \rightarrow$ $\operatorname{Max}(T V, T Y)$ are fibrations.

The proof of Theorem 4.1 is carried out by starting with Lemma4.3, applying Lemma 4.4 handle by handle, and an inverse limit argument. We refer the reader to $[\mathrm{P}$ for the details. Applying the inverse limit argument in the proof of Theorem 4.1 and 4.2, we have the following.

Lemma 4.5. Let $W$ be a codimension 0 compact submanifold of an open manifold $X$. Then, the restriction maps $\rho: \operatorname{Sbm}(X, Y) \rightarrow \operatorname{Sbm}(\mathrm{W}, \mathrm{Y})$ and $\rho: \operatorname{Max}(T X, T Y) \rightarrow \operatorname{Max}(T W, T Y)$ are fibrations.

To be precise, in the literature an open manifold could have a non-empty boundary. Thus, the following lemma might be in fact contained in Lemma 4.5. however, we give it here as a precise statement we need in the proof of Theorem A,

Lemma 4.6. Suppose that $X$ is a manifold with no compact component and $\partial X \neq \emptyset$. Let $W$ be a codimension 0 compact submanifold of $X$ such that $\partial X \subset \partial W$. Then, the restriction maps $\rho: \operatorname{Sbm}(X, Y) \rightarrow \operatorname{Sbm}(\mathrm{W}, \mathrm{Y})$ and $\rho: \operatorname{Max}(T X, T Y) \rightarrow \operatorname{Max}(T W, T Y)$ are fibrations.

Now, we can prove Theorem A, We add a correct consideration on the trivialization of the tangent bundles, however, we mostly follow the proof in My.

Proof of Theorem A. Assume that (11) holds. Then the preimage by $\varphi$ of a semiline starting from the origin to the end of $\mathbb{R}^{2}$ is a surface in $M$ which is 
bounded by $\varphi^{-1}(0)=L$. By the condition of $\varphi$ on the transverse orientation to $L$, we may choose the orientation on the surface so that $L$ represents the null-class in the locally finite homology group. Moreover, as mentioned in the Introduction, $\varphi$ determines a trivialization of $T M$ which restricts to a tangential framing of $L$. The projection with respect to the framing associated with this tangential framing of $L$ coincides with $\varphi$ near $L$. Thus, (21) holds.

Next, assume that (2) holds. Choose a framing $\nu: \bigsqcup_{j=1}^{n}\left(S^{1} \times D^{2}\right)_{j} \rightarrow$ $N(L)$ which is preferred and suppose there exists a trivialization of $T M$ which restricts to the trivialization of $T N(L)$ determined by the tangential framing induced by $\nu$. For $0 \leq r \leq 1$, set $D^{2}(r):=\{z \in \mathbb{C}|| z \mid \leq r\}$ and $N_{r}(L):=\nu\left(\bigsqcup_{j=1}^{n}\left(S^{1} \times D^{2}(r)\right)_{j}\right)$. Define $\pi: N(L) \rightarrow D^{2} \subset \mathbb{C}=\mathbb{R}^{2}$ to be the composition pr $\circ \nu^{-1}$, where pr : $\bigsqcup_{j=1}^{n}\left(S^{1} \times D^{2}\right)_{j} \rightarrow D^{2}$ is the natural projection onto a single disk. Set $X:=M \backslash \operatorname{Int} N_{1 / 2}(L)$ and $W:=$ $N(L) \backslash \operatorname{Int} N_{1 / 2}(L)$. Note that $\partial X=\partial N_{1 / 2}(L)$ since $\partial M=\emptyset$.

We consider the following commutative diagram consisting of the differential maps $d$ and the restriction maps $\rho$.

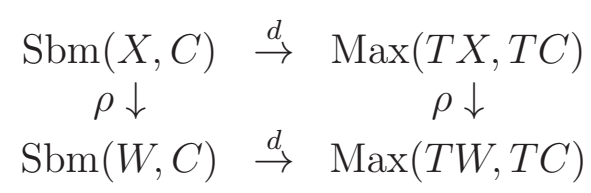

where $C$ denotes $\mathbb{R}^{2} \backslash \operatorname{Int} D^{2}\left(\frac{1}{2}\right)$. In the diagram the horizontal arrows are weak homotopy equivalences by Theorem 4.2 and 4.1, and the vertical arrows are fibrations by Lemma 4.6. The projection $\pi: N(L) \rightarrow D^{2}$ restricted to $W$, denoted by $\pi \mid W$, belongs to $\operatorname{Sbm}(W, C)$. For $d(\pi \mid W) \in \operatorname{Max}(T W, T C)$, we have an extension as follows.

Claim. There exists $\Phi \in \operatorname{Max}(T X, T C)$ such that $\rho(\Phi)=d(\pi \mid W)$.

Proof. By the canonical trivialization of $T \mathbb{R}^{2}$, we may consider that $T C=$ $C \times \mathbb{R}^{2}$. By the assumption, we have a trivialization of $T X=T M \mid X$ which restricts to the trivialization of $T W=T N(L) \mid W$ determined by the framing $\nu$. Thus, $d(\pi \mid W)$ is represented as

$$
T W \cong W \times \mathbb{R}^{3} \rightarrow C \times \mathbb{R}^{2} \cong T C ;\left(x,\left(v_{1}, v_{2}, v_{3}\right)\right) \mapsto\left(\pi(x),\left(v_{2}, v_{3}\right)\right)
$$


Therefore, in order to obtain an extension of $d(\pi \mid W)$, we only have to show that the map $\pi \mid W: W \rightarrow C$ extends to $X$. For this purpose, we may consider the problem up to homotopy. Since $W$ (resp. $C$ ) is homotopy equivalent to $\partial N_{1 / 2}(L)=\partial X$ (resp. $S^{1}$ ), the projection $\pi \mid W$ determines a homotopy class $[\pi \mid W] \in\left[\partial X, S^{1}\right]$. We will give an extension of $[\pi \mid W]$ in $\left[X, S^{1}\right]$. Since $S^{1}$ is the Eilenberg-MacLane space $K(\mathbb{Z}, 1)$, there are natural bijections $\left[X, S^{1}\right] \rightarrow H^{1}(X ; \mathbb{Z})$ and $\left[\partial X, S^{1}\right] \rightarrow H^{1}(\partial X ; \mathbb{Z})$ which commute the restriction maps (cf. Spanier [S]). Combining these maps with PoincaréLefschetz duality (see Massey [Ms] for the locally finite homology version), we have the following sign-commutative diagram.



Here, the horizontal rows are cohomology and homology exact sequences and the vertical arrows are Poincaré-Lefschetz duality isomorphisms. As noted above, we may consider that $[\pi \mid W]$ belongs to $\left[\partial X, S^{1}\right]=H^{1}(\partial X ; \mathbb{Z})$. We claim that $\delta[\pi \mid W]=0$. Through the Poincaré-Lefschetz duality $[\pi \mid W]$ corresponds to the homology class represented by the fiber of $\pi$ in $H_{1}(\partial X ; \mathbb{Z})$. Consequently the class $[\pi \mid W]$ corresponds to the class represented by the union of longitudes of $N(L)$. Since the longitudes are preferred, the class vanishes in $H_{1}^{\infty}(X ; \mathbb{Z})$ which implies that $\delta[\pi \mid W]=0$. Hence, by the exactness of the sequence, we have a class in $H^{1}(X ; \mathbb{Z})=\left[X, S^{1}\right]$ which restricts to $[\pi \mid W]$.

Remark 4.1. This extension lemma does not hold under the condition of the original incorrect theorem in $\mathrm{My}$. In fact, the consideration of framings of the tangent bundles was necessary.

In the diagram (11) the differential map $d: \operatorname{Sbm}(X, C) \rightarrow \operatorname{Max}(T X, T C)$ is a weak homotopy equivalence. Therefore, there exists $\psi \in \operatorname{Sbm}(X, C)$ such that $d \psi$ is homotopic to $\Phi$ in $\operatorname{Max}(T X, T C)$. Thus $d \rho(\psi)=\rho(d \psi)$ is homotopic to $d(\pi \mid W)$ in $\operatorname{Max}(T W, T C)$. Since the differential map is a weak 
homotopy equivalence, $\rho(\psi)$ and $\pi \mid W$ are regularly homotopic. Moreover, the restriction map $\rho: \operatorname{Sbm}(X, C) \rightarrow \operatorname{Sbm}(W, C)$ is a fibration, thus the regular homotopy from $\rho(\psi)$ to $\pi \mid W$ is covered by a regular homotopy of $\psi$. Hence we conclude that there exists $\varphi \in \operatorname{Sbm}(X, C)$ whose restriction to $W$ is $\pi \mid W$. This completes the proof of Theorem $\mathrm{A}$.

Finally, we give the proof of Theorem B. In the proof below, the description of cutting open the residual components is improved in comparison with the proof in $\mathrm{My}$.

Proof of Theorem $B$. Let $L$ be any $n$-component link in $M$. Choose a framing $\nu: \bigsqcup_{j=1}^{n}\left(S^{1} \times D^{2}\right)_{j} \rightarrow N(L)$. By twisting the framing of a component once in the meridional direction if necessary, we may assume that there exists a trivialization of $T M$ whose restriction to $N(L)$ is equal to the trivialization induced by the chosen framing of $L$. Let $\pi: N(L) \rightarrow D^{2}$ be the projection defined as in the proof of Theorem A. Now we consider the following commutative diagram.

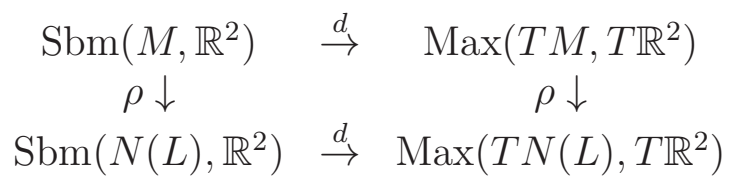

Here, the restriction maps $\rho$ are fibrations by Lemma 4.5 and the differential maps $d$ are weak homotopy equivalences by Theorem 4.2 and 4.1 . We claim the existence of an extension of $d \pi$.

Claim. There exists $\Phi \in \operatorname{Max}\left(T M, T \mathbb{R}^{2}\right)$ such that $\rho(\Phi)=d \pi$.

Proof of Claim. Since the trivialization of $T M \mid N(L)$ induced by the chosen framing of $L$ is the restriction of a trivialization of $T M$, as in the proof of the claim in the proof of Theorem $\mathrm{A}$, we only have to show that the map $\pi$ extends to $M$ up to homotopy. However, since $\mathbb{R}^{2}$ is contractible this is clear.

Now, chasing the diagram (3) in the same way as in the proof of Theorem A shows that there exists an extension $\hat{\varphi} \in \operatorname{Sbm}\left(M, \mathbb{R}^{2}\right)$ of $\pi$. If the union of compact components of $\hat{\varphi}^{-1}(0)$ is equal to $L$, then set $\varphi:=\hat{\varphi}$ and we are done. Otherwise, let $R$ denote the union of compact components of 
$\hat{\varphi}^{-1}(0)$ which are not contained in $L$. Note that $R$ has at most countably infinitely many components. We will cut open these residual circles $R$ by curves ending to ends of $M$. It suffices to consider the case that there are infinitely many components of $R$. The proof in the case of only finitely many components is similar and simpler. Note that the components of $R$ cannot accumulate. Now we fix an increasing filtration by codimension 0 compact connected submanifolds $N_{k}$ of $M\left(k \in \mathbb{Z}_{\geq 0}\right)$ such that $\cup_{k=0}^{\infty} N_{k}=M$. (We may assume that $M$ is connected.) Suppose that we choose a decreasing filtration by open subsets $U_{k}^{e}$, each of which is a component of $M \backslash N_{k}$ for $k \in \mathbb{Z}_{\geq 0}$. Then it defines an end $e$ of $M$. Here, we assume that $\partial N_{k} \cap R=\emptyset$ for any $k \in \mathbb{Z}_{\geq 0}$. Let $\mathcal{E}$ denote the subset of the end set of $M$ consisting of all ends $e=\left\{U_{k}^{e}\right\}_{k \in \mathbb{Z}_{\geq 0}}$ such that $U_{k}^{e} \cap R \neq \emptyset$ for any $k \in \mathbb{Z}_{\geq 0}$. Since $\mathcal{E}$ is at most a countable set, we index it by natural numbers: $\mathcal{E}=\left\{e_{m}\right\}_{m \in \mathbb{N}}$. Also, since there are at most countably many components of $R$, we number them as follows. First, number the components of $R \cap N_{0}$ as $R_{1} \sqcup R_{2} \sqcup \cdots \sqcup R_{\ell_{0}}$, next $R \cap\left(N_{1} \backslash \operatorname{Int} N_{0}\right)=R_{\ell_{0}+1} \sqcup \cdots \sqcup R_{\ell_{1}}$, and inductively $R \cap\left(N_{k} \backslash \operatorname{Int} N_{k-1}\right)=$ $R_{\ell_{k-1}+1} \sqcup \cdots \sqcup R_{\ell_{k}}$ for $k \in \mathbb{N}$.

We then define, inductively, simple curves $\alpha_{m}:[0, \infty) \rightarrow M\left(m \in \mathbb{Z}_{\geq 0}\right)$ which cut $R$ open. First, for the end $e_{1}=\left\{U_{k}^{1}\right\}_{k \in \mathbb{Z}_{\geq 0}} \in \mathcal{E}$, the sequence of the components of $R \cap\left(\cup_{k=0}^{\infty}\left(U_{k}^{1} \cap N_{k+1}\right)\right)$ is an infinite subsequence of the components of $R$, which tends to the end $e_{1}$. Then we choose a simple curve $\alpha_{1}$ in $\cup_{k=0}^{\infty}\left(U_{k}^{1} \cap N_{k+1}\right)$ which passes through one point in each circle of $R \cap\left(\cup_{k=0}^{\infty}\left(U_{k}^{1} \cap N_{k+1}\right)\right)$ and tends to $e_{1}$. Here, we choose $\alpha_{1}$ so that it passes through $R_{\ell}$ in order with respect to the indices $\ell$ of the circle $R_{\ell}$. Set $R^{(1)}:=R \cap\left(\cup_{k=0}^{\infty}\left(U_{k}^{1} \cap N_{k+1}\right)\right)$. Inductively, for the end $e_{m}=\left\{U_{k}^{m}\right\}_{k \in \mathbb{Z}_{\geq 0}} \in \mathcal{E}$, the sequence of the components of $R^{(m)}:=\left(R \backslash \cup_{i=1}^{m-1} R^{(i)}\right) \cap\left(\cup_{k=0}^{\infty}\left(U_{k}^{m} \cap N_{k+1}\right)\right)$ is an infinite subsequence of circles of $R$ and we choose a simple curve $\alpha_{m}$ in $\cup_{k=0}^{\infty}\left(U_{k}^{m} \cap N_{k+1}\right)$ which passes through $R^{(m)}$ in order and tends to $e_{m}$. Moreover, we choose all the curves $\alpha_{m}$ so that they do not intersect $L$ and are mutually disjoint. Note that $R^{(0)}:=R \backslash \cup_{m=1}^{\infty} R^{(m)}$ is compact. Thus, the components of $R^{(0)}$ are finitely many circles and we can easily choose a simple curve $\alpha_{0}$ which passes through those circles and tends to an end of 
M. As is similar to the case of $\alpha_{m}$ above, we take $\alpha_{0}$ so that it does not intersect $L$ nor $\alpha_{m}(m \in \mathbb{N})$. Now we claim the following.

Claim. $\left(M \backslash \cup_{m=0}^{\infty} \operatorname{Im}\left(\alpha_{m}\right), L\right)$ is diffeomorphic to $(M, L)$.

Proof. Set $P:=D^{2} \times[0, \infty)$ and let $\alpha:[0, \infty) \rightarrow P$ be the curve defined by $\alpha(t):=(0, t+1)$. Then we can easily construct a diffeomorphism between $P$ and $P \backslash \operatorname{Im}(\alpha)$ which is the identity near the boundary $\left(D^{2} \times\{0\}\right) \cup\left(\partial D^{2} \times\right.$ $[0, \infty))$. By the construction of $\alpha_{m}$ the set $\left\{\alpha_{m}(0)\right\}$ is discrete in $M$ and the curves $\left\{\alpha_{m}\right\}$ do not accumulate. Hence, the claim follows.

Setting $\varphi:=\hat{\varphi} \mid\left(M \backslash \cup_{m=0}^{\infty} \operatorname{Im}\left(\alpha_{m}\right)\right)$, we have the desired submersion. This completes the proof of Theorem B

Acknowledgements. The author would like to express his hearty gratitude to Gilbert Hector who informed him that his earlier work contained an error. He also thanks Daniel Peralta-Salas for studying, with G. Hector, the problem which the author considered before and was caused by the author's misunderstanding. Without their notice the author would never have found the correction and new results in the point of view of the author.

\section{REFERENCES}

[HP] G. Hector, D. Peralta-Salas, Integrable embeddings and foliations, Amer. J. Math. 134 (2012), 773-825.

[Ms] W. S. Massey, Homology and cohomology theory, Marcel Dekker Inc., 1978.

[My] S. Miyoshi, Links and globally completely integrable vector fields on an open 3manifold, Topology 34 (1995) 383-387.

[P] A. Phillips, Submersions of open manifolds, Topology 6 (1966) 171-295.

[R] D. Rolfsen, Knots and Links, AMS Chelsea publ., 1976.

[S] E. H. Spanier, Algebraic Topology, Springer, 1995.

[W] J. H. C. Whitehead, The immersion of an open 3-manifold in Euclidean 3-space, Proc. London Math. Soc. 11 (1961) 81-90.

Department of Mathematics, Chuo University, 1-13-27 Kasuga Bunkyo-Ku, TOKYO, 112-8551, JAPAN

E-mail address: miyoshi@math.chuo-u.ac.jp 